\title{
A novel contactless technique for thermal field mapping and thermal conductivity determination: Two-Laser Raman Thermometry
}

Cite as: Rev. Sci. Instrum. 85, 034901 (2014); https://doi.org/10.1063/1.4867166

Submitted: 28 December 2013 . Accepted: 16 February 2014 . Published Online: 10 March 2014

J. S. Reparaz, E. Chavez-Angel, M. R. Wagner, B. Graczykowski, J. Gomis-Bresco, F. Alzina, and C. M. Sotomayor Torres

ARTICLES YOU MAY BE INTERESTED IN

Reduction of the thermal conductivity in free-standing silicon nano-membranes investigated by non-invasive Raman thermometry

APL Materials 2, 012113 (2014); https://doi.org/10.1063/1.4861796

Nanoscale thermal transport. II. 2003-2012

Applied Physics Reviews 1, 011305 (2014); https://doi.org/10.1063/1.4832615

Nanoscale thermal transport

Journal of Applied Physics 93, 793 (2003); https://doi.org/10.1063/1.1524305

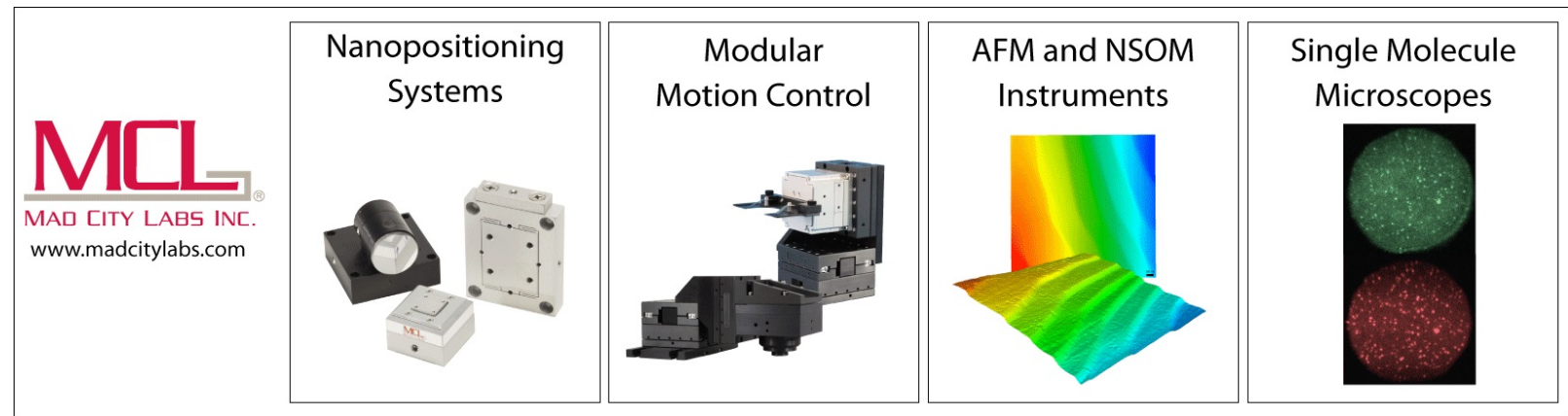




\title{
A novel contactless technique for thermal field mapping and thermal conductivity determination: Two-Laser Raman Thermometry
}

\author{
J. S. Reparaz, ${ }^{1}$ E. Chavez-Angel, ${ }^{1,2}$ M. R. Wagner, ${ }^{1}$ B. Graczykowski, ${ }^{1}$ J. Gomis-Bresco, ${ }^{1}$ \\ F. Alzina, ${ }^{1}$ and C. M. Sotomayor Torres ${ }^{1,3}$ \\ ${ }^{1}$ ICN2 - Institut Catala de Nanociencia i Nanotecnologia, Campus UAB, 08193 Bellaterra, Barcelona, Spain \\ ${ }^{2}$ Department of Physics, UAB, 08193 Bellaterra, Barcelona, Spain \\ ${ }^{3}$ ICREA, Passeig Lluís Companys 23, 08010 Barcelona, Spain
}

(Received 28 December 2013; accepted 16 February 2014; published online 10 March 2014)

\begin{abstract}
We present a novel contactless technique for thermal conductivity determination and thermal field mapping based on creating a thermal distribution of phonons using a heating laser, while a second laser probes the local temperature through the spectral position of a Raman active mode. The spatial resolution can be as small as $300 \mathrm{~nm}$, whereas its temperature accuracy is $\pm 2 \mathrm{~K}$. We validate this technique investigating the thermal properties of three free-standing single crystalline Si membranes with thickness of 250,1000 , and $2000 \mathrm{~nm}$. We show that for two-dimensional materials such as freestanding membranes or thin films, and for small temperature gradients, the thermal field decays as $T(r) \propto \ln (r)$ in the diffusive limit. The case of large temperature gradients within the membranes leads to an exponential decay of the thermal field, $T \propto \exp [-A \cdot \ln (r)]$. The results demonstrate the full potential of this new contactless method for quantitative determination of thermal properties. The range of materials to which this method is applicable reaches far beyond the here demonstrated case of $\mathrm{Si}$, as the only requirement is the presence of a Raman active mode. () 2014 AIP Publishing LLC. [http://dx.doi.org/10.1063/1.4867166]
\end{abstract}

\section{INTRODUCTION}

A precise determination of the thermal conductivity $(\kappa)$ of a given material is usually a difficult task since the heat per unit time flowing in a certain spatial direction $\left(Q \equiv P_{a b s}\right)$ must be precisely determined. Contrary to the analogous case of electrons (or holes) propagating in a metal, where the electrical current can be easily measured using galvanometer through the magnetic field created by the current, there is no direct method to measure heat currents. Instead, the heat flux per unit time is usually inferred considering the geometry of the system and the excitation source, the temperature gradient $(\Delta T)$ is measured in the heat flow direction and, finally, the thermal conductivity is obtained through Fourier's law $\left(\kappa=P_{a b s} / \Delta T\right)$. Several electrical and optical techniques have been developed to measure the thermal conductivity of a large variety of materials and structures. ${ }^{1-13}$ Optical methods have recently attracted considerable attention since most of them are contactless and, thus, require few sample preparation. Such techniques can be divided into two main categories: (i) steady-state techniques, ${ }^{3-8}$ and (ii) transient techniques. ${ }^{9-13}$ An advantage of transient techniques is that they do not require the knowledge of the absorbed power in the sample since they are only sensitive to the thermal diffusivity, $\alpha=\kappa / \rho C_{p}$ (where $\rho$ is the density and $C_{p}$ the specific heat), which is usually proportional to a characteristic decay time of the system. On the contrary, steady-state techniques provide a direct determination of the thermal conductivity provided that the power absorbed in the sample is known. Depending on the materials and structures under investigation either of these approaches might be more convenient.

Here we present a novel contactless technique suitable for thermal conductivity determination and thermal mapping of one- and two-dimensional structures provided that the samples exhibit a detectable Raman signal of any of its active modes, and a sufficiently large spectral shift as function of temperature. The thermal conductivity is obtained by fitting the spatial decay of the thermal field using a diffusive model based on Fourier's law. We provide the solution of the two-dimensional steady-state heat equation for an isotropic medium for the cases where the thermal conductivity is constant $\left(\kappa_{0}\right)$ or varies with temperature $(\kappa(T))$. The thermal properties of three free-standing Si membranes with thicknesses of 250,1000 , and $2000 \mathrm{~nm}$ were studied to validate the present approach.

\section{DESCRIPTION OF THE TECHNIQUE: TWO-LASER RAMAN THERMOMETRY}

We have developed a contactless technique suitable to measure the thermal conductivity of nanowires, free-standing membranes, thin films on a given substrate, and bulk samples. This technique is based on previous works ${ }^{5,14-19}$ which used the temperature dependent Raman spectra of a given material to obtain its thermal conductivity (commonly known as Raman thermometry). Briefly, the temperature rise $\left(T_{\max }\right)$ at the focused laser spot, which depends on the incident laser power, is obtained from the spectral position of any Raman mode, provided that a previous calibration of the Raman shift with temperature has been made. Subsequently, the steadystate heat equation is solved analytically or numerically, depending on the geometry and dimensionality of the problem, to obtain the relationship between $\kappa, T_{\max }$ and the absorbed power on the sample $\left(P_{a b s}\right)$. The main drawback of this technique is that only one point of the temperature field is probed 
to determine $\kappa$ since the heating and the probe lasers are the same. We recall that for the case of diffusive thermal transport at least two points are required to obtain the temperature field and, thus, $\kappa$. To fulfill this requirement an assumption must be made, i.e., at sufficiently large distances from the laser spot the temperature equals that of the thermal bath $\left(T_{b a t h}\right)$. Thus, the two points required to obtain the temperature field are: $\left\{T=T_{\text {max }} ; \mathbf{r}=0\right\}$ and $\left\{T=T_{\text {bath }} ; \mathbf{r} \rightarrow \infty\right\}$. Although in some cases this approximation is valid such as, e.g., in the case of bulk samples, in most cases it fails leading to an inaccurate determination of $\kappa$.

The Two-Laser Raman Thermometry (2LRT) technique overcomes this drawback by mapping with a low power probe laser the entire thermal field temperature distribution created by a high power laser impinging on the sample. In order to apply this technique, the material under investigation ought to meet the following requirements:

- Temperature sensor: A Raman active mode must be detectable in order to probe the local temperature. This mode will ideally exhibit a strong temperature dependence $(\partial \omega / \partial T)$ to be a good temperature sensor. A calibration of the Raman shift versus temperature must be known in advance.

- Excitation source: The sample should have a reasonable absorbance in the spectral region of the heating laser to set up the temperature distribution. The absorbed power $\left(P_{a b s}\right)$ needs to be determined for each experimental conditions.

- Dimensionality: The sample must be of one- or twodimensions with lateral size larger than the optical resolution $(\approx 300 \mathrm{~nm})$. It is also possible to study three-dimensional structures provided that the heat distribution has a spherical symmetry with respect to the heating laser spot.

The main advantage of this technique as compared to other contactless steady-state methods, such as infrared thermometry, is its sub-micrometer spatial resolution. Techniques based on scanning probes such as Scanning Thermal Microscopy (SThM), or Infrared Near Field Scanning Optical Microscopy (IR-NSOM) also provide high spatial resolution, but their main drawback is the rather cumbersome tip calibration. On the other hand, time-resolved techniques such as Time-Domain Thermoreflectance (TDTR) or Thermal Transient Grating (TTG) only give indirect access to $\kappa$ through the thermal diffusivity $\left(\alpha=\kappa / \rho C_{P}\right)$. In particular, we mention a recent work by Schmotz et $a{ }^{3}{ }^{3}$ where the authors measure temperature maps of a $\mathrm{Si}$ membrane by tracking the local change in the absorption coefficient, which exhibit a Fabry-Pérot interference behavior due to the sub-wavelength thickness of the membranes. Although this technique is extremely accurate regarding temperature determination accurate $(\delta T<1 \mathrm{mK})$, it is limited to semitransparent samples, thin enough to produce an interference pattern. The two-laser Raman thermometry technique reported here is perhaps a more general approach since it is based on the Raman effect, which is determined by atomic vibrations within a unit cell.

In Fig. 1(a) we show schematically the 2LRT experimental arrangement. A heating laser with $\lambda_{1}=405 \mathrm{~nm}$ is focused onto the lower surface of the Si membranes, whereas a probe laser with $\lambda_{2}=488 \mathrm{~nm}$ is scanned over its upper surface to obtain the local temperature. We note that while relatively high powers were used for the heating laser $\left(\lambda_{1}\right)$ in order to create a spatially dependent thermal field, low powers were employed for the probe laser $\left(\lambda_{2}\right)$ to avoid an additional thermal perturbation. We typically used a 10:1 ratio between the heating and probe laser powers. Both lasers are focused onto the samples using long distance $50 \times$ objectives with numerical aperture of NA $=0.55$. Although these objectives provide lower spatial resolution than short distances objectives, with typically $\mathrm{NA} \geq 0.7$, they allow to perform the thermal maps in a controlled environment or under variable temperature conditions. Figure 1(b) displays a two-dimensional (2D) temperature map of a $250 \mathrm{~nm}$ thick Si membrane obtained using 2LRT. The maximum temperature at the center, i.e., with the heating and probe lasers at the same position of the membrane, is $\approx 800 \mathrm{~K}$ and a radially symmetric thermal decay is observed in a constant temperature projection plane. The radial symmetry observed in the 2D maps arises from the isotropic thermal behavior of $\mathrm{Si}$ at room temperature. ${ }^{20}$ However, for materials with a spatially dependent thermal conductivity $\left(\kappa_{i j}\right)$, an (a)

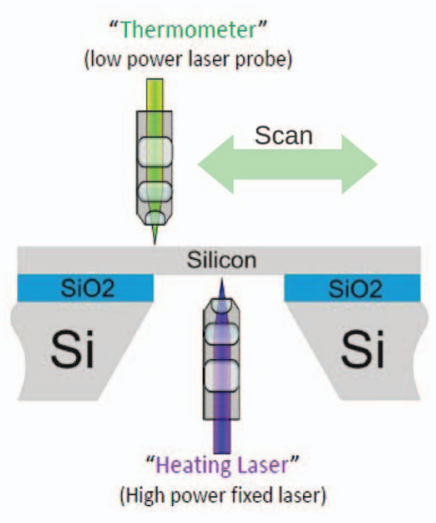

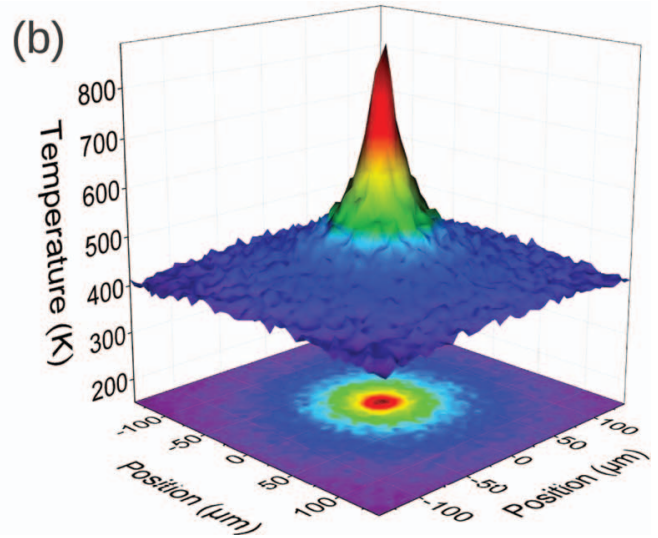

FIG. 1. (a) Schematics of the two-laser Raman thermometry experimental setup. The lower laser is used as heating source, whereas the upper laser probes the local temperature through the spectral shift of the longitudinal optical Raman mode of Si. (b) Two-dimensional thermal map of a $250 \mathrm{~nm}$ thick free-standing Si membrane. A projection of the thermal field is also shown in a lower plane. 
asymmetric thermal decay is expected. The symmetric heat distribution observed in Fig. 1 justifies the assumption made to derive Eqs. (1) and (2) and reduces the measurement to only one line scan on the $(X, Y, 0)$ plane containing the ori$\operatorname{gin}(X, Y, Z)=(0,0,0)$. It is noteworthy that the temperature field does not fully decay to the thermal bath temperature of $294 \mathrm{~K}$, and instead reaches only $\approx 400 \mathrm{~K}$ at $150 \mu \mathrm{m}$ from the heating laser. The origin of this slow thermal decay is the relatively high thermal conductivity of this membrane, as well as the purely diffusive thermal transport regime. As shown by Eq. (1), or simply by Fourier's law, a larger thermal conductivity implies that heat distributes more efficiently through the material, thus leading to smaller temperature gradients. We note that a ballistic transport regime would account for a faster thermal decay as shown in Ref. 26.

Taking into account the geometry of the measurements in Fig. 1 we derive the analytical solution for the temperature field in the case of a free-standing isotropic membrane. A temperature distribution is created upon excitation with a point like laser source as shown in Fig. 1. The solution is simply given by integrating Fourier's equation: $P_{a b s} / A=-\kappa \nabla T$, where $P_{a b s}$ is the power injected in the system, $A$ is the cross sectional area of the heat flux, $\kappa$ is the thermal conductivity, and $T$ is the temperature. The cross sectional area depends on the geometry of the heat flux and is $A=2 \pi r d$ for freestanding thin membranes with a point-like heating source, where $d$ is the thickness of the membrane:

$$
\begin{gathered}
T(r)=T_{0}-\frac{P_{a b s}}{2 \pi d \kappa_{0}} \ln \left(r / r_{0}\right) \rightarrow \kappa=\kappa_{0}, \\
T(r)=T_{0}\left(\frac{r}{r_{0}}\right)^{-P_{a b s} / 2 \pi d a} \rightarrow \kappa=\frac{a}{T},
\end{gathered}
$$

where we have considered a temperature independent thermal conductivity $\left(\kappa_{0}\right)$ as well as a $1 / T$ behavior $(\kappa(T))$, which is typical for most semiconductors at high temperatures. ${ }^{25}$ The constant $a$ can be simply written as $a=294 \cdot \kappa(T=294)$. We note that the spatial distribution of the heat source, i.e., a Gaussian heat source, can be considered as $P=\int_{s p o t} P \mid r$ $-r^{\prime} \mid d r^{\prime}$, but we avoid this approach since it does not provide any benefits and obscures the simplicity of Eqs. (1) and (2). In the present case of Si use as thermometer the temperature dependent Raman shift of the zone center longitudinal optical (LO) phonon at $\omega_{0}=520 \mathrm{~cm}^{-1}$ (at $300 \mathrm{~K}$ ). Fig. 2(a) shows typical Raman spectra of the $250 \mathrm{~nm}$ thick membrane at different relative positions between the heating and probe lasers. We recall that typically first order Raman modes in semiconductors exhibit a redshift with increasing temperatures with temperature coefficients ranging between 20 and $65 \mathrm{~K} / \mathrm{cm}^{-1}$. $^{21-24}$ The Si LO peak gradually redshifts and broadens as the position of the probe laser is closer to the heating laser, i.e., smaller values of $r$, indicating an increase of the local temperature. The accuracy of the temperature determination is given by the spectral resolution. In our case, the resolving power of the spectrometer used is about $0.4 \mathrm{~cm}^{-1}$, whereas the spectral resolution resulting from fits to the spectra using a Lorentzian function is $0.05 \mathrm{~cm}^{-1}$. In Fig. 2(b) we show the calibration between Raman shift and temperature as obtained using a cryostat to control the bath

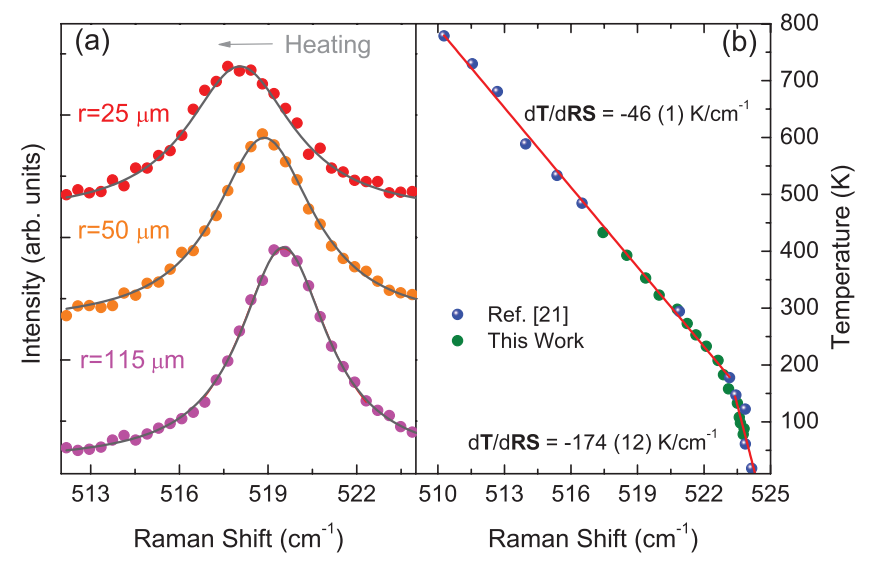

FIG. 2. (a) Representative Raman spectra recorded at different distances $(r)$ from the heating laser. The solid lines are least squares fits using Lorentzian functions. (b) Calibration of the Raman shift versus temperature. The blue dots were extracted from Ref. 21.

temperature as well as the data from Ref. 21. A linear relation is found between 200 and $800 \mathrm{~K}$ with a slope $d T / d R S$ $=-46(1) \mathrm{K} / \mathrm{cm}^{-1}$, which leads to a temperature accuracy of $\pm 2 \mathrm{~K}$ considering the spectral resolution. The temperature regime $T<150 \mathrm{~K}$ was also fitted using a linear function obtaining $d T / d R S=-174(12) \mathrm{K} / \mathrm{cm}^{-1}$.

\section{RESULTS AND DISCUSSION}

In order to obtain the thermal conductivity of the membranes we measured a line scan of the thermal field in the $(X, Y, 0)$ plane containing the coordinates origin as shown in Fig. 3(a) for three Si membranes with thicknesses of 250 , 1000 , and $2000 \mathrm{~nm}$. The membranes were single crystalline with a surface roughness $\mathrm{R}_{a}=0.2 \mathrm{~nm}$ and $\mathrm{rms}=0.15 \mathrm{~nm}$, and were purchased from NORCADA Inc. The inset shows an optical image of the $250 \mathrm{~nm}$ thick membrane. All three membranes exhibit a qualitatively similar behavior, i.e., a similar decay length and half width of the thermal field profile. We note that the minimum temperature obtained in these maps is also above the thermal bath temperature, reaching $\approx 330 \mathrm{~K}$ at $150 \mu \mathrm{m}$ in comparison to the $\approx 400 \mathrm{~K}$ obtained in the $2 \mathrm{D}$ thermal map of Fig. 1(b), which arises from the smaller heating powers used for the line scans of Fig. 3. This is also reflected in the maximum temperature rise observed at the central position of the line scans. According to Eq. (1) the thermal decay is linear in $\ln (r)$, thus, we show in Fig. 3(b) the data corresponding to Fig. 3(a) in logarithmic scale. As predicted by Eq. (1) the thermal field decays linearly with a slope given by $\partial T(r) / \partial \ln (r)=P_{a b s} / 2 \pi d \kappa_{0}$. The different slopes in Fig. 3(b) simply result from different combinations of $P_{a b s}, d$, and $\kappa_{0}$ with no scaling law involving $d$ or $\kappa_{0}$. In fact, normalization of the temperature rise, $T_{\text {rise }}=T(r)-T_{\text {bath }}=T(r)-294 \mathrm{~K}$, results in equal slopes for the three membranes as predicted by Eq. (1) (see inset of Fig. 3(b)). Finally, using Eq. (1) we calculate the thermal conductivity of each membrane obtained from the slopes fitted to the data in Fig. 3(b). We obtain $\kappa=80$ $\pm 3,133 \pm 6,147 \pm 8 \mathrm{~W} / \mathrm{mK}$ for the 250,1000 , and $2000 \mathrm{~nm}$ thick membranes, respectively, which are in excellent agreement with the results reported in Refs. 16 and 27-30. 
(a)

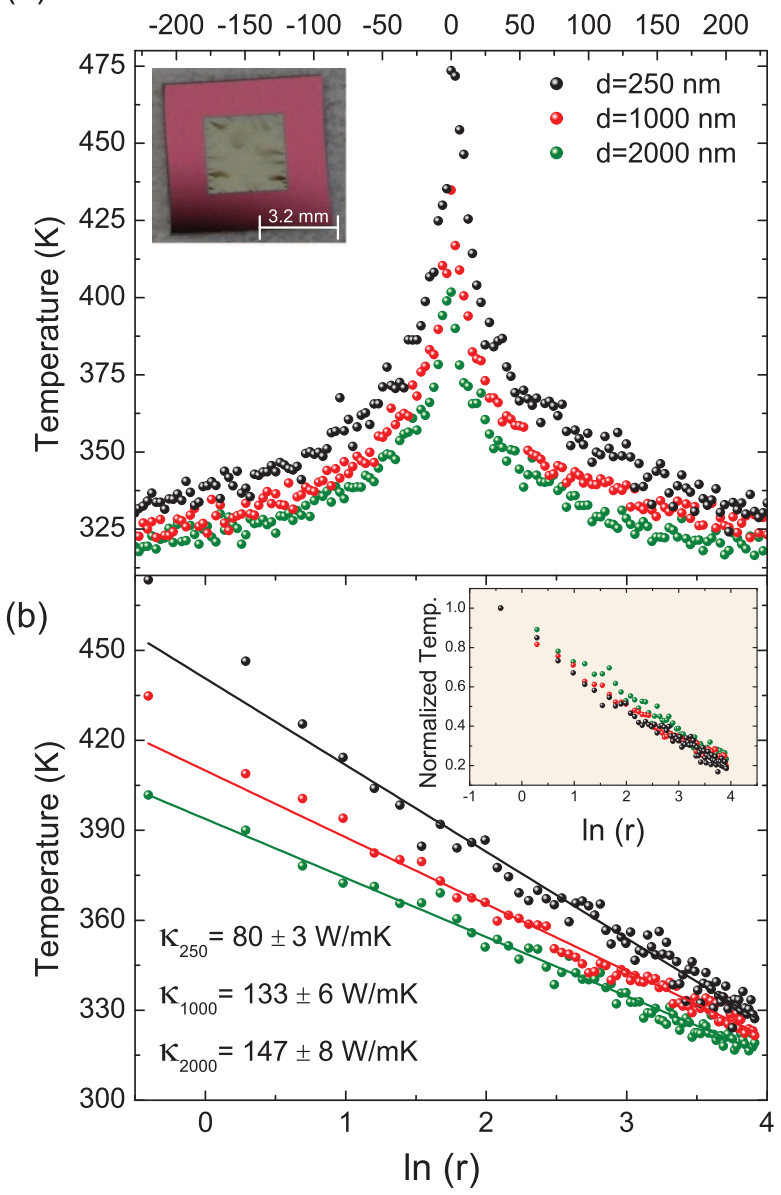

FIG. 3. (a) Line scans of the thermal field as shown in Fig. 1(b). The line scan was recorded using a lower heating power than for the 2D map in Fig. 1(b) to ensure that $\kappa \neq \kappa(T)$. The inset shows an optical image of the $250 \mathrm{~nm}$ thick membrane. (b) Same as (a) but in logarithmic scale to visualize the $\ln (\mathrm{r})$ dependence as predicted by Eq. (1). The inset shows the normalized temperature rise, $T_{\text {rise }}=T(r)-294 \mathrm{~K}$ for the three membranes.

The influence of a temperature dependent thermal conductivity on the line shape of the thermal field is given by Eq. (2). The typical temperature dependence of the thermal conductivity for most semiconductors at high temperatures $(T$ $>200 \mathrm{~K})$ is $\kappa(T)=a / T^{n}$, where $a$ is a material dependent constant and $n$ is usually close to 1 . Herein, we assume the case $n=1$ for simplicity since our main purpose is to show the effect of temperature on the decay of the thermal field. Figure 4 displays several line scans using different heating powers for the $250 \mathrm{~nm}$ thick membrane. The maximum temperature rise gradually increases with increasing heating power as predicted by Eqs. (1) and (2). Once more, a qualitatively similar line width and decay of the thermal field profile as function of the heating power is observed in Fig. 4(a). However, a detailed analysis of the thermal decay exposes the dependence predicted by Eq. (2) as we show in Fig. 4(b). We recall that in logarithmic scale and for low heating powers, i.e., $\kappa=\kappa_{0}$ $\neq \kappa(T)$, the thermal field decays as $T \propto \ln (r)$ as shown by Eq. (1), whereas considering $\kappa(T)=a / T$, an exponential dependence on distance $T \propto \exp [-A \cdot \ln (r)]$ is expected. In fact, this is clearly observed in Fig. 4(b) with increasing heating (a)

(b)

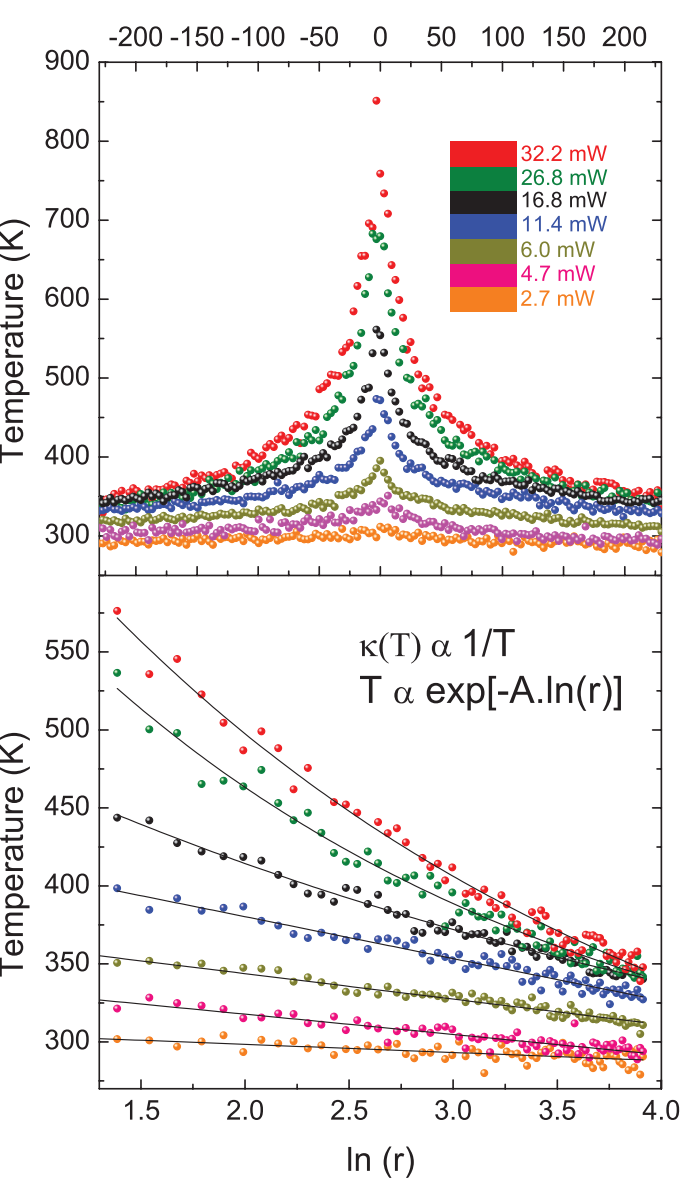

FIG. 4. (a) Thermal line scans using the different heating powers for the $250 \mathrm{~nm}$ thick membrane. (b) Same as (a) but in logarithmic scale to observe the deviation from the $T \propto \ln (r)$ behavior given by Eq. (1).

power. The thermal field gradually deviates from a straight line in logarithmic scale, $\ln (r)$, towards an exponential behavior. In this case, it is still possible to determine the thermal conductivity using Eq. (2) although the error increases substantially due to the small curvature $\left(A=P_{a b s} / 2 \pi d a\right)$ of the thermal decay. Although a clear deviation from a linear behavior is observed as the heating power increases, this effect is negligible at low heating powers and the thermal decay can be fully explained using Eq. (1).

The main potential of the technique reported here is demonstrated in Figs. 3 and 4. The determination of the thermal conductivity reduces simply a fit to a straight line in logarithmic scale at low heating powers, which substantially reduces the error. In addition, assuming that the functional dependence of the thermal conductivity on temperature is known, the thermal conductivity can be determined over a wide temperature range. This technique is suitable to investigate a large variety of materials given that they exhibit appreciable Raman signal. In addition to one- and twodimensional materials it is also possible to extend it to the three-dimensional case provided a spherical symmetry around the heating spot is maintained. Furthermore, this technique can be extended to the case of thin films supported on substrates, although a numerical analysis may be necessary to solve the heat equation. 


\section{CONCLUSIONS}

We have developed a novel contactless technique for thermal conductivity determination and thermal field mapping based on the concept of Raman thermometry. A two-laser approach is used to create and probe the spatial decay of the thermal field from which the thermal conductivity is extracted. This technique is suitable to investigate the temperature distribution in one- and two-dimensional structures, and it can be extended to three-dimensions for surface temperature mapping. The temperature resolution depends on the investigated material and it is $\pm 2 \mathrm{~K}$ for $\mathrm{Si}$, whereas its spatial resolution is diffraction limited and can be as low as $300 \mathrm{~nm}$.

The simplest solution for the temperature distribution in the isotropic two-dimensional case with a temperature independent thermal conductivity is shown to be $T(r) \propto \ln (r)$, and $\partial T(r) / \partial \ln (r)=-P_{a b s} / 2 \pi d \kappa_{0}$. Thus, the thermal conductivity can be determined by fitting the slope of the spatial decay of the thermal field. More sophisticated models considering $\kappa(T)$ are possible leading to an exponential decay of the temperature field $T(r) \propto \exp [-A \cdot \ln (r)]$ and were experimentally demonstrated heating the membranes up to $800 \mathrm{~K}$.

Finally, we describe the conditions that must be fulfilled by the sample under investigation in order to apply this technique. This technique should provide an extra step towards a deeper understanding of thermal management in $n$ dimensional materials since it gives the complete thermal response of a system, $T(r)$, subjected to a thermal perturbation.

\section{ACKNOWLEDGMENTS}

The authors acknowledge the financial support from the FP7 projects MERGING (Grant No. 309150), NANO-RF (Grant No. 318352), NANOTHERM (Grant No. 318117), NANOTEG-ENIAC (Grant No. 270789-2), and the Spanish MICINN projects nanoTHERM (Grant No. CSD2010-0044) and TAPHOR (MAT2012-31392). E.C.A. gratefully acknowledges a Becas Chile 2010 CONICYT fellowship from the Chilean government.

${ }^{1}$ D. G. Cahill, Rev. Sci. Instrum. 61, 802 (1990).

${ }^{2}$ I. Hatta, Y. Sasuga, R. Kato, and A. Maesono, Rev. Sci. Instrum. 56, 1643 (1985).
${ }^{3}$ M. Schmotz, P. Bookjans, E. Scheer, and P. Leiderer, Rev. Sci. Instrum. 81, 114903 (2010).

${ }^{4}$ C. B. Saltonstall, J. Serrano, P. M. Norris, P. E. Hopkins, and T. E. Beechem, Rev. Sci. Instrum. 84, 064903 (2013).

${ }^{5}$ R. Tsu and J. G. Hernandez, Appl. Phys. Lett. 41, 1016 (1982).

${ }^{6}$ C. C. Williams and H. K. Wickramasinghe, Appl. Phys. Lett. 49, 1587 (1986).

${ }^{7}$ S. Govorkov, W. Ruderman, M. W. Horn, R. B. Goodmann, and M. Rothschild, Rev. Sci. Instrum. 68, 3828 (1997).

${ }^{8}$ A. J. Schmidt, R. Cheaito, and M. Chiesa, Rev. Sci. Instrum. 80, 094901 (2009).

${ }^{9}$ W. J. Parker, R. J. Jenkins, C. P. Butler, and G. L. Abbott, J. Appl. Phys. 32, 1679 (1961).

${ }^{10}$ C. A. Paddock and G. L. Eesley, J. Appl. Phys. 60, 285 (1986).

${ }^{11}$ A. Harata, H. Nishimura, and T. Sawada, Appl. Phys. Lett. 57, 132 (1990).

${ }^{12}$ W. S. Capinski and H. J. Maris, Rev. Sci. Instrum. 67, 2720 (1996).

${ }^{13}$ J. A. Johnson, A. A. Maznev, M. T. Bulsara, E. A. Fitzgerald, T. C. Harman, S. Calawa, C. J. Vineis, G. Turner, and K. A. Nelson, J. Appl. Phys. 111, 023503 (2012).

${ }^{14}$ S. Périchon, V. Lysenko, B. Remaki, D. Barbier, and B. Champagnon, J. Appl. Phys. 86, 4700 (1999).

${ }^{15}$ S. Périchon, V. Lysenko, P. Roussel, B. Remaki, B. Champagnon, D. Barbier, and P. Pinard, Sens. Actuators A 85, 335 (2000).

${ }^{16}$ X. Liu, X. Wu, and T. Ren, Appl. Phys. Lett. 98, 174104 (2011).

${ }^{17}$ S. Huang, X. Ruan, J. Zou, X. Fu, and H. Yang, Microsyst. Technol. 15, 837 (2009).

${ }^{18}$ E. Chávez-Ángel, J. S. Reparaz, J. Gomis-Bresco, M. R. Wagner, J. Cuffe, B. Graczykowski, A. Shchepetov, H. Jiang, M. Prunnila, J. Ahopelto, F. Alzina, and C. M. S. Torres, APL Mater. 2, 012113 (2014).

${ }^{19}$ A. A. Balandin, S. Ghosh, W. Bao, I. Calizo, D. Teweldebrhan, F. Miao, and C. N. Lau, Nano Lett. 8, 902 (2008).

${ }^{20}$ At low temperatures ballistic heat transport occurs and, thus, Eqs. (1) and (2) are not valid. Furthermore, heat transport becomes highly asymmetric in $\mathrm{Si}$ as demonstrated by phonon focusing through heat pulse experiments. However, at sufficiently large temperatures the thermal behavior is purely diffusive and $\mathrm{Si}$ behaves as thermally isotropic.

${ }^{21}$ J. Menéndez and M. Cardona, Phys. Rev. B 29, 2051 (1984).

${ }^{22}$ I. Calizo, A. A. Balandin, W. Bao, F. Miao, and C. N. Lau, Nano Lett. 7, 2645 (2007).

${ }^{23}$ G. Irmer, M. Wenzel, and J. Monecke, Phys. Stat. Sol. B 195, 85 (1996).

${ }^{24}$ M. Liu, L. Bursill, S. Prawer, and R. Beserman, Phys. Rev. B 61, 3391 (2000).

${ }^{25}$ C. J. Glassbrenner and G. A. Slack, Phys. Rev. 134, A1058 (1964).

${ }^{26}$ J. T. Karvonen, T. Kühn, and I. J. Maasilta, Chin. J. Phys. 49, 435 (2011).

${ }^{27}$ M. Asheghi, Y. K. Leung, S. S. Wong, and K. E. Goodson, Appl. Phys. Lett. 71, 1798 (1997).

${ }^{28}$ M. Asheghi, M. N. Touzelbaev, K. E. Goodson, Y. K. Leung, and S. S. Wong, J. Heat Transfer 120, 30 (1998).

${ }^{29}$ Y. S. Ju and K. E. Goodson, Appl. Phys. Lett. 74, 3005 (1999).

${ }^{30}$ W. Liu and M. Asheghi, J. Appl. Phys. 98, 123523 (2005). 